\title{
Non-relativistic gravity theory based on an enlargement of the extended Bargmann algebra
}

\author{
Patrick Concha $^{a}$ and Evelyn Rodríguez ${ }^{b}$ \\ ${ }^{a}$ Instituto de Física, Pontificia Universidad Católica de Valparaíso, \\ Casilla 4059, Valparaiso, Chile \\ ${ }^{b}$ Departamento de Ciencias, Facultad de Artes Liberales, Universidad Adolfo Ibáñez, \\ Viña del Mar, Chile \\ E-mail: patrick.concha@pucv.cl, evelyn.rodriguez@edu.uai.cl
}

ABSTRACT: In this work we study a non-relativistic three dimensional Chern-Simons gravity theory based on an enlargement of the Extended Bargmann algebra. A finite nonrelativistic Chern-Simons gravity action is obtained through the non-relativistic contraction of a particular U(1) enlargement of the so-called AdS-Lorentz algebra. We show that the non-relativistic gravity theory introduced here reproduces the Maxwellian Exotic Bargmann gravity theory when a flat limit $\ell \rightarrow \infty$ is applied. We also present an alternative procedure to obtain the non-relativistic versions of the AdS-Lorentz and Maxwell algebras through the semigroup expansion method.

Keywords: Chern-Simons Theories, Field Theories in Lower Dimensions, Gauge Symmetry, Space-Time Symmetries

ARXiv EPrint: 1906.00086 


\section{Contents}

1 Introduction $\quad 1$

2 Three-dimensional AdS-Lorentz Chern-Simons gravity, U(1) enlargements and flat limit

2.1 AdS-Lorentz Chern-Simons gravity and flat limit 2

$2.2 \mathrm{U}(1)$ enlargements 5

3 Non-relativistic Chern-Simons AdS-Lorentz gravity $\quad 6$

3.1 Contraction process and enlarged extended Bargmann algebra 6

3.2 Non-relativistic Chern-Simons action 8

4 Non-relativistic algebras and S-expansion $\quad 10$

$\begin{array}{ll}\text { 4.1 Maxwellian exotic Bargmann algebra } & 10\end{array}$

$\begin{array}{lll}4.2 & \text { Enlargement of the extended Bargmann algebra } & 12\end{array}$

$\begin{array}{lll}5 & \text { Discussion } & 14\end{array}$

\section{Introduction}

There has been a renewed interest in non-relativistic (NR) theories due to their utilities to approach strongly coupled condensed matter systems [1-8] as well as NR effective field theories [9-12]. At the gravity level, the study of NR gravitational theories has been discussed by diverse authors in [13-28]. A NR theory can be obtained by a suitable limiting process from a relativistic theory. In particular, through this work, the NR limit corresponds to the limit in which the speed of light $c$ tends to infinity. It is well known that in such NR limit, the AdS spacetime becomes the Newton-Hooke symmetry which in the flat limit corresponds to the Galilei symmetry [29-36].

On the other hand, three-dimensional gravity models are interesting and simple toy models as they can be formulated as a Chern-Simons (CS) gauge theory [37-39]. In particular, CS approach allows us to construct diverse relativistic and non-relativistic (super)gravity actions. Nevertheless, the construction of a NR CS action might lead to infinities and degeneracy. Such difficulties can be overcome by enlarging the field content of the relativistic theory $[24,25,40,41]$. In the case of the relativistic Poincaré CS gravity theory the NR limit requires to introduce two central extension in order to admit a non-degenerate bilinear form. Such extension leads to the exotic Bargmann algebra which allows us to define a proper finite NR CS action [25, 26].

Recently, the NR limit of a three-dimensional CS gravity theory based on a particular deformation and enlargement of the Poincaré symmetry was presented in [42]. Such symmetry, known as the Maxwell algebra, has been introduced in [43-45] to describe the presence 
of a constant electromagnetic field background in a Minkowski space. Interestingly, the infinities and degeneracy are avoided by considering the NR contraction of a [Maxwell] $\oplus u(1) \oplus u(1) \oplus u(1)$ algebra leading to the Maxwellian Exotic Bargmann (MEB) algebra.

In this work, we explore the NR limit of a three-dimensional CS gravity theory based on the AdS-Lorentz algebra. Such symmetry is a semi-simple enlargement of the Poincaré one and has been initially introduced in [46, 47]. The AdS-Lorentz algebra and its generalizations have been particularly useful to recover (pure) Lovelock gravity theories from CS and Born-Infeld theories [48-50]. At the supersymmetric level, the supersymmetric extension of the AdS-Lorentz algebra has been used to introduce alternatively a cosmological constant term in four-dimensional supergravity [51-54]. More recently, a BMS-like ansatz for a three-dimensional CS theory based on the AdS-Lorentz algebra has been presented in [55]. In particular, the asymptotic symmetry at null infinity turns out to be a semisimple enlargement of the $\mathfrak{b m s}_{3}$ algebra. An interesting feature of the AdS-Lorentz algebra is that it reproduces the Maxwell algebra through a flat limit $\ell \rightarrow \infty$.

Here we present a NR CS gravity based on a particular enlargement of the extended Bargmann (EEB) algebra by considering the NR contraction of the [AdS-Lorentz] $\oplus u(1) \oplus$ $u(1) \oplus u(1)$ algebra. Such $U(1)$ enlargement not only allows us to construct a finite NR CS action but also to obtain the MEB gravity in the flat limit. We also present an alternative procedure to obtain the EEB and MEB algebras through the semigroup expansion mechanism ( $S$-expansion) [56-58]. Such procedure note only provides us with the commutators of the NR algebras but also gives the non-vanishing components of the invariant tensor which are crucial to the proper construction of a CS action.

The paper is organized as follows: in section 2, we give a brief review of the threedimensional relativistic AdS-Lorentz CS gravity theory. The section 3 is devoted to the NR contraction process of the AdS-Lorentz gravity theory. In section 4, we present an alternative mechanism to obtain the EEB and MEB algebras by considering the $S$-expansion method. Section 5 is devoted to discussion and possible developments.

Note added: while this manuscript was in the process of typesetting, it came to our knowledge the ref. [59], which possesses some overlap with particular cases of our results.

\section{Three-dimensional AdS-Lorentz Chern-Simons gravity, U(1) enlarge- ments and flat limit}

\subsection{AdS-Lorentz Chern-Simons gravity and flat limit}

In this section, we review the construction of a three-dimensional CS gravity based on a semi-simple enlargement of the Poincaré group. The mentioned group is known as the AdSLorentz group [46, 60, 61] and the corresponding algebra is a deformation and enlargement of the AdS algebra. The commutators of the AdS-Lorentz algebra read

$$
\begin{array}{rlrl}
{\left[J_{A}, J_{B}\right]} & =\epsilon_{A B C} J^{C}, & {\left[P_{A}, P_{B}\right]} & =\epsilon_{A B C} Z^{C}, \\
{\left[J_{A}, Z_{B}\right]} & =\epsilon_{A B C} Z^{C}, & {\left[Z_{A}, Z_{B}\right]=\frac{1}{\ell^{2}} \epsilon_{A B C} Z^{C},} \\
{\left[J_{A}, P_{B}\right]=\epsilon_{A B C} P^{C},} & {\left[Z_{A}, P_{B}\right]=\frac{1}{\ell^{2}} \epsilon_{A B C} P^{C},}
\end{array}
$$


where $J_{A}$ are the spacetime rotations, $P_{A}$ are the spacetime translations and $Z_{A}$ are nonAbelian generators. Note that $A, B, C=0,1,2$ are the Lorentz indices which are lowered and raised with the Minkowski metric $\eta_{A B}=(-1,1,1)$ and $\epsilon_{A B C}$ is the Levi Civita tensor which is normalized as $\epsilon_{012}=-\epsilon^{012}=1$. Let us note that the name "AdS-Lorentz" is due to the fact that the algebra $(2.1)$ can be written as the direct sum $\mathfrak{s o}(2,2) \oplus \mathfrak{s o}(2,1)$ by a suitable redefinition of the generators,

$$
\begin{aligned}
J_{A} & =\ell^{2} \hat{Z}_{A}, \\
P_{A} & =\hat{P}_{A}, \\
Z_{A} & =\hat{J}_{A}-\ell^{2} \hat{Z}_{A} .
\end{aligned}
$$

Let us construct now the relativistic CS action for the AdS-Lorentz symmetry. The three-dimensional CS action is given by

$$
I[A]=\int\left\langle A d A+\frac{2}{3} A^{3}\right\rangle,
$$

where $A$ is the one-form gauge connection and $\langle\cdots\rangle$ denotes the invariant trace. In particular, the one-form $A$ taking values in the AdS-Lorentz algebra (2.1) reads

$$
A=W^{A} J_{A}+E^{A} P_{A}+K^{A} Z_{A}
$$

where $W^{A}$ is the one-form spin connection, $E^{A}$ is the vielbein and $K^{A}$ is the gauge field along the non-Abelian generator $Z_{A}$. The associated curvature two-form is

$$
F=R^{A}(W) J_{A}+R^{A}(E) P_{A}+R^{A}(K) Z_{A},
$$

where

$$
\begin{aligned}
R^{A}(W) & :=d W^{A}-\frac{1}{2} \epsilon^{A B C} W_{B} W_{C}, \\
R^{A}(E) & :=D_{W} E^{A}-\frac{1}{\ell^{2}} \epsilon^{A B C} K_{B} E_{C}, \\
R^{A}(K) & :=D_{W} K^{A}-\frac{1}{2 \ell^{2}} \epsilon^{A B C} K_{B} K_{C}-\frac{1}{2} \epsilon^{A B C} E_{B} E_{C} .
\end{aligned}
$$

In particular, the Lorentz covariant derivative is defined as $D_{W} \Theta^{A}:=d \Theta^{A}-\epsilon^{A B C} W_{B} \Theta_{C}$. The non-vanishing components of an invariant tensor of rank 2 for the AdS-Lorentz algebra are given by $[55,60,62]$

$$
\begin{aligned}
\left\langle J_{A} J_{B}\right\rangle & =\mu_{0} \eta_{A B}, & \left\langle P_{A} P_{B}\right\rangle & =\frac{\mu_{2}}{\ell^{2}} \eta_{A B}, \\
\left\langle J_{A} P_{B}\right\rangle & =\frac{\mu_{1}}{\ell} \eta_{A B}, & \left\langle Z_{A} Z_{B}\right\rangle & =\frac{\mu_{2}}{\ell^{4}} \eta_{A B}, \\
\left\langle J_{A} Z_{B}\right\rangle & =\frac{\mu_{2}}{\ell^{2}} \eta_{A B}, & \left\langle Z_{A} P_{B}\right\rangle & =\frac{\mu_{1}}{\ell^{3}} \eta_{A B},
\end{aligned}
$$

where the arbitrary constants $\mu_{0}, \mu_{1}$ and $\mu_{2}$ can be redefined as

$$
\mu_{0} \rightarrow \alpha_{0}, \quad \mu_{1} \rightarrow \alpha_{1} \ell, \quad \mu_{2} \rightarrow \alpha_{2} \ell^{2} .
$$


In this way the flat limit $\ell \rightarrow \infty$ is well defined and (2.6) with the redefinition (2.7) leads to the invariant tensor of the Maxwell group in three-dimensions. Note that applying this limit in (2.1) leads us to the commutation relations of the Maxwell algebra. As we will see in the next sections, this behaviour also arises at the level of the non-relativistic gravities.

Considering the AdS-Lorentz connection one-form (2.4) and the invariant tensor (2.6) with the redefinition (2.7) in the general form (2.3), we find that the explicit form of the relativistic CS gravity action invariant under the AdS-Lorentz symmetry reads

$$
\begin{aligned}
& I_{R}=\int\left[\alpha_{0}\left(W^{A} d W_{A}+\frac{1}{3} \epsilon^{A B C} W_{A} W_{B} W_{C}\right)\right. \\
& +\alpha_{1}\left(2 E_{A} R^{A}(W)+\frac{2}{\ell^{2}} E_{A} F^{A}(K)+\frac{1}{3 \ell^{2}} \epsilon^{A B C} E_{A} E_{B} E_{C}\right) \\
& \left.+\alpha_{2}\left(T^{A} E_{A}+\frac{1}{\ell^{2}} \epsilon^{A B C} E_{A} K_{B} E_{C}+2 K_{A} R^{A}(W)+\frac{2}{\ell^{2}} K_{A} D_{W} K^{A}+\frac{1}{3 \ell^{4}} \epsilon^{A B C} K_{A} K_{B} K_{C}\right)\right],
\end{aligned}
$$

where we have defined the torsion two-form $T^{A}:=D_{W} E^{A}$, and the curvature $F^{A}(K):=$ $D_{W} K^{A}-\frac{1}{2 \ell^{2}} \epsilon^{A B C} K_{B} K_{C}$. From (2.8) we see that the action is split into three different sectors proportional to the $\alpha^{\prime}$ s constants. The gravitational CS term is related to the $\alpha_{0}$ constant, while the EH term plus a cosmological constant term appear along the $\alpha_{1}$ constant together with a term depending on the gauge field $K^{A}$. The last term proportional to $\alpha_{2}$ contain a torsional term plus couplings of the gravitational gauge fields with the $K^{A}$ field. It is important to point out that each of the three sectors is invariant under the AdS-Lorentz symmetry. In particular, the infinitesimal gauge transformations $\delta_{\Lambda} A=d \Lambda+[A, \Lambda]$, with gauge parameter $\Lambda=\rho^{A} J_{A}+\varepsilon^{A} P_{A}+\gamma^{A} Z_{A}$, are given by

$$
\begin{aligned}
\delta_{\Lambda} W^{A} & =D_{W} \rho^{A} \\
\delta_{\Lambda} E^{A} & =D_{W} \varepsilon^{A}-\epsilon^{A B C} \rho_{B} E_{C}-\frac{1}{\ell^{2}} \epsilon^{A B C}\left(K_{B} \varepsilon_{C}-\gamma_{B} E_{C}\right), \\
\delta_{\Lambda} K^{A} & =D_{W} \gamma^{A}-\epsilon^{A B C}\left(E_{B} \varepsilon_{C}-\rho_{B} K_{C}\right)-\frac{1}{\ell^{2}} \epsilon^{A B C} K_{B} \gamma_{C} .
\end{aligned}
$$

The CS action (2.8) is invariant, modulo boundary terms, under these gauge transformations.

Note that the flat limit $\ell \rightarrow \infty$ applied to (2.8) leads to the relativistic CS action for the Maxwell symmetry in three-dimensions [42, 63, 64]. In the same way, this limit leads to the gauge transformations for the Maxwell symmetry when is considered in (2.9).

The field equations coming from (2.8) are given by

$$
\begin{aligned}
\delta W^{A}: & 0=\alpha_{0} R_{A}(W)+\alpha_{1}\left(T_{A}-\frac{1}{\ell^{2}} \epsilon_{A B C} K^{B} E^{C}\right)+\alpha_{2}\left(F_{A}(K)-\frac{1}{2} \epsilon_{A B C} E^{B} E^{C}\right), \\
\delta E^{A}: & 0=\alpha_{1}\left(R_{A}(W)+\frac{1}{\ell^{2}} F_{A}(K)-\frac{1}{2 \ell^{2}} \epsilon_{A B C} E^{B} E^{C}\right)+\alpha_{2}\left(T_{A}-\frac{1}{\ell^{2}} \epsilon_{A B C} K^{B} E^{C}\right), \\
\delta K^{A}: & 0=\frac{\alpha_{1}}{\ell^{2}}\left(T_{A}-\frac{1}{\ell^{2}} \epsilon_{A B C} K^{B} E^{C}\right)+\alpha_{2}\left(R_{A}(W)+\frac{1}{\ell^{2}} F_{A}(K)-\frac{1}{2 \ell^{2}} \epsilon_{A B C} E^{B} E^{C}\right),
\end{aligned}
$$


which can be written as

$$
\begin{aligned}
R_{A}(W) & =0, \\
T_{A}-\frac{1}{\ell^{2}} \epsilon_{A B C} K^{B} E^{C} & =0, \\
F_{A}(K)-\frac{1}{2} \epsilon_{A B C} E^{B} E^{C} & =0 .
\end{aligned}
$$

As is expected, when $\ell \rightarrow \infty$ in (2.10) the equations lead to the Maxwell field equations.

Let us now study the NR limit of the presented theory. Here we are interested in the limit where the speed of light is taken to infinity. It is very well-known that taking the limit $c \rightarrow \infty$ in the relativistic Lagrangian, might lead to infinities. In order to cancel the divergences, one can include extra fields in the relativistic theory. As we are also interested in finding the NR Maxwell CS gravity [42] in the limit $\ell \rightarrow \infty$, we will consider the [AdSLorentz] $\oplus u(1) \oplus u(1) \oplus u(1)$ theory as our initial relativistic theory, i.e, we add three new extra fields to the field content. In this way, the NR contraction should lead to a finite Lagrangian and to a NR algebra with a non-degenerate bilinear form.

Before studying the non-relativistic limit of the AdS-Lorentz gravity theory, let us first introduce the extra $\mathrm{U}(1)$ gauge fields in the one-form gauge connection (2.4).

\section{$2.2 \quad \mathrm{U}(1)$ enlargements}

Motivated by the discussion of the previous section, we now include three extra U(1) oneforms gauge fields, $M, S$ and $T$ in (2.4) as

$$
A=W^{A} J_{A}+E^{A} P_{A}+K^{A} Z_{A}+M Y_{1}+S Y_{2}+T Y_{3} .
$$

The new Abelian generators satisfy the following non-zero invariant tensors

$$
\begin{array}{ll}
\left\langle Y_{1} Y_{1}\right\rangle=\alpha_{2}, & \left\langle Y_{2} Y_{3}\right\rangle=\alpha_{2}, \\
\left\langle Y_{2} Y_{2}\right\rangle=\alpha_{0}, & \left\langle Y_{3} Y_{3}\right\rangle=\frac{\alpha_{2}}{\ell^{2}}, \\
\left\langle Y_{1} Y_{2}\right\rangle=\alpha_{1}, & \left\langle Y_{1} Y_{3}\right\rangle=\frac{\alpha_{1}}{\ell^{2}} .
\end{array}
$$

Then, the non-vanishing components of the invariant tensor for the new [AdS-Lorentz] $\oplus u(1) \oplus u(1) \oplus u(1)$ algebra are given by (2.6) along with (2.12). Considering the new enlarged one-form gauge connection (2.11) and invariant tensor, the relativistic CS action is written as

$$
\begin{aligned}
I_{R}=\int[ & \alpha_{0}\left(W^{A} d W_{A}+\frac{1}{3} \epsilon^{A B C} W_{A} W_{B} W_{C}+S d S\right) \\
& +\alpha_{1}\left(2 E_{A} R^{A}(W)+\frac{2}{\ell^{2}} E_{A} F^{A}(K)+\frac{1}{3 \ell^{2}} \epsilon^{A B C} E_{A} E_{B} E_{C}+2 M d S+\frac{2}{\ell^{2}} M d T\right) \\
& +\alpha_{2}\left(T^{A} E_{A}+\frac{1}{\ell^{2}} \epsilon^{A B C} E_{A} K_{B} E_{C}+2 K_{A} R^{A}(W)+\frac{2}{\ell^{2}} K_{A} D_{W} K^{A}\right. \\
& \left.\left.+\frac{1}{3 \ell^{4}} \epsilon^{A B C} K_{A} K_{B} K_{C}+M d M+2 S d T+\frac{1}{\ell^{2}} T d T\right)\right]
\end{aligned}
$$


In the following we will show that the inclusion of these three extra $U(1)$ gauge fields allows to cancel the divergences that appear in the limiting process. Let us remark that in this work we are interested in the contraction of the [AdS-Lorentz] $\oplus u(1) \oplus u(1) \oplus u(1)$ algebra since, as we will show in the next section, the resulting NR algebra admits on one hand a non-degenerate invariant tensor, and on the other hand, leads to the MEB algebra introduced in [42] in the $\ell \rightarrow \infty$ limit.

\section{Non-relativistic Chern-Simons AdS-Lorentz gravity}

\subsection{Contraction process and enlarged extended Bargmann algebra}

In the previous section we have constructed an extended relativistic AdS-Lorentz algebra. Now, considering the Inönü-Wigner contraction of this algebra we will obtain a NR version of the AdS-Lorentz algebra. To this purpose, we will consider a dimensionless parameter $\xi$, and we will express the relativistic generators as a linear combination of new generators involving the $\xi$ parameter.

We define the contraction process through the identification of the relativistic generators defining the [AdS-Lorentz] $\oplus u(1) \oplus u(1) \oplus u(1)$ algebra, with the NR generators (denoted with a tilde) as

$$
\begin{aligned}
& J_{0}=\frac{\tilde{J}}{2}+\xi^{2} \tilde{S}, \quad J_{a}=\xi \tilde{G}_{a}, \quad Y_{2}=\frac{\tilde{J}}{2}-\xi^{2} \tilde{S}, \\
& P_{0}=\frac{\tilde{H}}{2 \xi}+\xi \tilde{M}, \quad P_{a}=\tilde{P}_{a}, \quad Y_{1}=\frac{\tilde{H}}{2 \xi}-\xi \tilde{M}, \\
& Z_{0}=\frac{\tilde{Z}}{2 \xi^{2}}+\tilde{T}, \quad Z_{a}=\frac{\tilde{Z}_{a}}{\xi}, \quad Y_{3}=\frac{\tilde{Z}}{2 \xi^{2}}-\tilde{T} .
\end{aligned}
$$

along with the following scaling

$$
\ell \rightarrow \xi \ell .
$$

This redefinition is required in order to have a well-defined limit $\xi \rightarrow \infty$. A particular enlargement of the extended Bargamm algebra, which we have denoted as EEB algebra, comes from the contraction of $(2.1)$ :

$$
\begin{aligned}
& {\left[\tilde{G}_{a}, \tilde{P}_{b}\right]=-\epsilon_{a b} \tilde{M}, \quad\left[\tilde{G}_{a}, \tilde{Z}_{b}\right]=-\epsilon_{a b} \tilde{T}, \quad\left[\tilde{H}, \tilde{Z}_{a}\right]=\frac{1}{\ell^{2}} \epsilon_{a b} \tilde{P}_{b},} \\
& {\left[\tilde{H}, \tilde{G}_{a}\right]=\epsilon_{a b} \tilde{P}_{b}, \quad\left[\tilde{J}, \tilde{Z}_{a}\right]=\epsilon_{a b} \tilde{Z}_{b}, \quad\left[\tilde{Z}_{a}, \tilde{Z}_{b}\right]=-\frac{1}{\ell^{2}} \epsilon_{a b} \tilde{T},} \\
& {\left[\tilde{J}, \tilde{P}_{a}\right]=\epsilon_{a b} \tilde{P}_{b}, \quad\left[\tilde{H}, \tilde{P}_{a}\right]=\epsilon_{a b} \tilde{Z}_{b}, \quad\left[\tilde{P}_{a}, \tilde{Z}_{b}\right]=-\frac{1}{\ell^{2}} \epsilon_{a b} \tilde{M},} \\
& {\left[\tilde{J}, \tilde{G}_{a}\right]=\epsilon_{a b} \tilde{G}_{b}, \quad\left[\tilde{P}_{a}, \tilde{P}_{b}\right]=-\epsilon_{a b} \tilde{T}, \quad\left[\tilde{Z}, \tilde{P}_{a}\right]=\frac{1}{\ell^{2}} \epsilon_{a b} \tilde{P}_{b},} \\
& {\left[\tilde{G}_{a}, \tilde{G}_{b}\right]=-\epsilon_{a b} \tilde{S}, \quad\left[\tilde{Z}, \tilde{G}_{a}\right]=\epsilon_{a b} \tilde{Z}_{b}, \quad\left[\tilde{Z}, \tilde{Z}_{a}\right]=\frac{1}{\ell^{2}} \epsilon_{a b} \tilde{Z}_{b} .}
\end{aligned}
$$

Here $a=1,2, \epsilon_{a b} \equiv \epsilon_{0 a b}, \epsilon^{a b} \equiv \epsilon^{0 a b}$. Such NR algebra contains three central extensions given by $\tilde{M}, \tilde{S}$ and $\tilde{T}$ which are related to the three extra $U(1)$ generators. Note that 
when we set $\tilde{Z}=\tilde{Z}_{a}=\tilde{T}=0$ the extended Bargmann algebra is recovered. On the other hand, in the $\ell \rightarrow \infty$ limit the EEB algebra leads to the MEB algebra [42].

It is interesting to note that the algebra (3.3) can be rewritten as three copies of the so-called Nappi-Witten algebra [65, 66],

$$
\begin{aligned}
{\left[\tilde{J}^{ \pm}, \tilde{G}_{a}^{ \pm}\right] } & =\epsilon_{a b} \tilde{G}_{b}^{ \pm}, & {\left[\tilde{G}_{a}^{ \pm}, \tilde{G}_{b}^{ \pm}\right] } & =-\epsilon_{a b} \tilde{S}^{ \pm} \\
{\left[\hat{\tilde{J}}, \hat{\tilde{G}}_{a}\right] } & =\epsilon_{a b} \hat{\tilde{G}}_{b}, & {\left[\hat{\tilde{G}}_{a}, \hat{\tilde{G}}_{b}\right] } & =-\epsilon_{a b} \hat{\tilde{S}}
\end{aligned}
$$

Such algebra can be viewed as a central extension of the homogeneous part of the Galilei algebra. Let us note that the three copies of the Nappi-Witten algebra reproduces the EEB algebra (3.3) by considering the following redefinitions

$$
\begin{aligned}
\tilde{G}_{a} & =\hat{\tilde{G}}_{a}+\tilde{G}_{a}^{+}+\tilde{G}_{a}^{-}, & \tilde{P}_{a} & =\frac{1}{\ell}\left(\tilde{G}_{a}^{+}-\tilde{G}_{a}^{-}\right), & \tilde{Z}_{a} & =\frac{1}{\ell^{2}}\left(\tilde{G}_{a}^{+}+\tilde{G}_{a}^{-}\right), \\
\tilde{S} & =\hat{\tilde{S}}+\tilde{S}^{+}+\tilde{S}^{-}, & \tilde{M} & =\frac{1}{\ell}\left(\tilde{S}^{+}-\tilde{S}^{-}\right), & \tilde{T} & =\frac{1}{\ell^{2}}\left(\tilde{S}^{+}+\tilde{S}^{-}\right), \\
\tilde{J} & =\hat{\tilde{J}}+\tilde{J}^{+}+\tilde{J}^{-}, & \tilde{H} & =\frac{1}{\ell}\left(\tilde{J}^{+}-\tilde{J}^{-}\right), & \tilde{Z} & =\frac{1}{\ell^{2}}\left(\tilde{J}^{+}+\tilde{J}^{-}\right) .
\end{aligned}
$$

A different redefinition of the generators allows us to rewrite the algebra (3.3) as the direct sum of the extended Newton-Hooke $\left\{\overline{\tilde{G}}_{a}, \overline{\tilde{P}}_{a}, \overline{\tilde{S}}, \overline{\tilde{M}}, \overline{\tilde{J}}, \overline{\tilde{H}}\right\}$ and the Nappi-Witten algebra $\left\{\overline{\tilde{Z}}_{a}, \overline{\tilde{T}}, \overline{\tilde{Z}}\right\}$. In fact, one could define

$$
\begin{array}{rlr}
\tilde{G}_{a}=\overline{\tilde{G}}_{a}+\overline{\tilde{Z}}_{a}, & \tilde{P}_{a}=\frac{\overline{\tilde{P}}_{a},}{\ell_{a}^{2}}, \\
\tilde{S}=\overline{\tilde{S}}+\overline{\tilde{T}}, & \tilde{M}=\overline{\tilde{M}}, & \tilde{T}=\frac{\tilde{\tilde{S}}}{\ell}, \\
\tilde{\ell^{2}}=\overline{\tilde{J}}+\overline{\tilde{Z}}, & \tilde{Z}=\frac{\tilde{\tilde{J}}}{\ell^{2}},
\end{array}
$$

such that the direct sum of the extended Newton-hooke and the Nappi-Witten algebra appears,

$$
\begin{array}{rlrl}
{\left[\overline{\tilde{G}}_{a}, \overline{\tilde{G}}_{b}\right]} & =-\epsilon_{a b} \overline{\tilde{S}}, & {\left[\overline{\tilde{J}}, \overline{\tilde{G}}_{a}\right]=\epsilon_{a b} \overline{\tilde{G}}_{b},} \\
{\left[\overline{\tilde{G}}_{a}, \overline{\tilde{P}}_{b}\right]=-\epsilon_{a b} \overline{\tilde{M}},} & {\left[\overline{\tilde{J}}, \overline{\tilde{P}}_{a}\right]=\epsilon_{a b} \overline{\tilde{P}}_{b},} \\
{\left[\overline{\tilde{P}}_{a}, \overline{\tilde{P}}_{b}\right]=-\frac{1}{\ell^{2}} \epsilon_{a b} \overline{\tilde{S}},} & {\left[\overline{\tilde{H}}, \overline{\tilde{G}}_{a}\right]=\epsilon_{a b} \overline{\tilde{P}}_{b},} \\
{\left[\overline{\tilde{H}}_{\overline{\tilde{P}}}\right]=\frac{1}{\ell^{2}} \epsilon_{a b} \overline{\tilde{G}}_{b},} & {\left[\overline{\tilde{Z}}, \overline{\tilde{Z}}_{a}\right]=\epsilon_{a b} \overline{\tilde{Z}}_{b} .}
\end{array}
$$

This is similar to the relativistic AdS-Lorentz algebra which can be written as the direct sum $\mathfrak{s o}(2,2) \oplus \mathfrak{s o}(2,1)$. In particular, the Nappi-Witten algebra is characterized by the presence of a central extension, denoted by $\tilde{\tilde{Z}}$. On the other hand, the Newton-Hooke symmetries have been recently studied in three-dimensional spacetime in $[32,33,36]$. The 
extended Newton-Hooke algebra (3.7), also known as exotic Newton-Hooke algebra [36] has two central extensions given by the generators $\overline{\tilde{S}}$ and $\overline{\tilde{M}}$. Such algebra is the NR version of the AdS algebra and appears as a NR contraction of a particular enlargement of the relativistic AdS algebra.

It is important to remark that, even though the NR algebra (3.7)-(3.8) looks simpler than (3.3), it is the basis $\left\{\tilde{J}, \tilde{G}_{a}, \tilde{H}, \tilde{P}_{a}, \tilde{Z}, \tilde{Z}_{a}, \tilde{S}, \tilde{M}, \tilde{T}\right\}$ which allows us to make contact with the Maxwellian Exotic Bargmann gravity through a proper flat limit. In addition, the relativistic AdS-Lorentz CS gravity theory has been studied previously in the literature [55, $62,67]$ in the basis $\left\{J_{A}, P_{A}, Z_{A}\right\}$ which satisfy $(2.1)$. It is then natural to construct the CS action related to the EEB algebra in the form (3.3).

\subsection{Non-relativistic Chern-Simons action}

Let us consider the explicit construction of a NR CS action based on the EEB algebra obtained in the previous section.

The non-vanishing components of a non degenerate invariant tensor are obtained from the contraction (3.1) of the relativistic invariant tensor (2.6) with the redefinition (2.7)

$$
\begin{aligned}
\langle\tilde{J} \tilde{S}\rangle & =-\tilde{\alpha}_{0}, \\
\left\langle\tilde{G}_{a} \tilde{G}_{b}\right\rangle & =\tilde{\alpha}_{0} \delta_{a b}, \\
\left\langle\tilde{G}_{a} \tilde{P}_{b}\right\rangle & =\tilde{\alpha}_{1} \delta_{a b}, \\
\langle\tilde{H} \tilde{S}\rangle & =\langle\tilde{M} \tilde{J}\rangle=-\tilde{\alpha}_{1}, \\
\left\langle\tilde{P}_{a} \tilde{P}_{b}\right\rangle & =\left\langle\tilde{G}_{a} \tilde{Z}_{b}\right\rangle=\tilde{\alpha}_{2} \delta_{a b}, \\
\langle\tilde{J} \tilde{T}\rangle & =\langle\tilde{H} \tilde{M}\rangle=\langle\tilde{S} \tilde{Z}\rangle=-\tilde{\alpha}_{2}, \\
\left\langle\tilde{Z}_{a} \tilde{Z}_{b}\right\rangle & =\frac{\tilde{\alpha}_{2}}{\ell^{2}} \delta_{a b}, \\
\left\langle\tilde{Z}_{a} \tilde{P}_{b}\right\rangle & =\frac{\tilde{\alpha}_{1}}{\ell^{2}} \delta_{a b}, \\
\langle\tilde{Z} \tilde{M}\rangle & =\langle\tilde{T} \tilde{H}\rangle=-\frac{\tilde{\alpha}_{1}}{\ell^{2}} \delta_{a b}, \\
\langle\tilde{Z} \tilde{T}\rangle & =-\frac{\tilde{\alpha}_{2}}{\ell^{2}} \delta_{a b},
\end{aligned}
$$

where the relativistic parameters $\alpha$ 's were rescaled as

$$
\alpha_{0}=\tilde{\alpha}_{0} \xi^{2}, \quad \alpha_{1}=\tilde{\alpha}_{1} \xi, \quad \alpha_{2}=\tilde{\alpha}_{2},
$$

in order to have a finite CS action. As is expected, the flat limit $\ell \rightarrow \infty$ applied to (3.9)(3.10) leads to the NR invariant tensors for the MEB algebra [42].

Now we can write the NR one-form gauge connection $\tilde{A}$ in terms of the NR generators as follows

$$
\tilde{A}=\tau \tilde{H}+e^{a} \tilde{P}_{a}+\omega \tilde{J}+\omega^{a} \tilde{G}_{a}+k \tilde{Z}+k^{a} \tilde{Z}_{a}+m \tilde{M}+s \tilde{S}+t \tilde{T}
$$


The NR curvature two-form is then

$$
\begin{aligned}
\tilde{F}= & R(\tau) \tilde{H}+R^{a}\left(e^{b}\right) \tilde{P}_{a}+R(\omega) \tilde{J}+R^{a}\left(\omega^{b}\right) \tilde{G}_{a}+R(k) \tilde{Z} \\
& +R^{a}\left(k^{b}\right) \tilde{Z}_{a}+R(m) \tilde{M}+R(s) \tilde{S}+R(t) \tilde{T},
\end{aligned}
$$

where

$$
\begin{aligned}
R(\tau) & =d \tau, \\
R^{a}\left(e^{b}\right) & =d e^{a}+\epsilon^{a c} \omega e_{c}+\epsilon^{a c} \tau \omega_{c}+\frac{1}{\ell^{2}} \epsilon^{a c} k e_{c}+\frac{1}{\ell^{2}} \epsilon^{a c} \tau k_{c}, \\
R(\omega) & =d \omega \\
R^{a}\left(\omega^{b}\right) & =d \omega^{a}+\epsilon^{a c} \omega \omega_{c}, \\
R(k) & =d k \\
R^{a}\left(k^{b}\right) & =d k^{a}+\epsilon^{a c} \omega k_{c}+\epsilon^{a c} \tau e_{c}+\epsilon^{a c} k \omega_{c}+\frac{1}{\ell^{2}} \epsilon^{a c} k k_{c}, \\
R(m) & =d m+\epsilon^{a c} e_{a} \omega_{c}+\frac{1}{\ell^{2}} \epsilon^{a c} e_{a} k_{c}, \\
R(s) & =d s+\frac{1}{2} \epsilon^{a c} \omega_{a} \omega_{c}, \\
R(t) & =d t+\epsilon^{a c} \omega_{a} k_{c}+\frac{1}{2} \epsilon^{a c} e_{a} e_{c}+\frac{1}{2 \ell^{2}} \epsilon^{a c} k_{a} k_{c} .
\end{aligned}
$$

Finally, the NR CS action invariant under the EEB algebra is

$$
\begin{array}{r}
I_{N R}=\int \tilde{\alpha}_{0}\left[\omega_{a} R^{a}\left(\omega^{b}\right)-2 s R(\omega)\right]+\tilde{\alpha}_{1}\left[2 e_{a} R^{a}\left(\omega^{b}\right)-2 m R(\omega)-2 \tau R(s)+\frac{2}{\ell^{2}} e_{a} F^{a}\left(k^{b}\right)\right. \\
\left.-\frac{2}{\ell^{2}} \tau R(t)-\frac{2}{\ell^{2}} m R(k)\right]+\tilde{\alpha}_{2}\left[e_{a} R^{a}\left(e^{b}\right)+k_{a} R^{a}\left(\omega^{b}\right)+\frac{1}{\ell^{2}} k_{a} R^{a}\left(k^{b}\right)\right. \\
\left.+\omega_{a} R^{a}\left(k^{b}\right)-2 s R(k)-2 m R(\tau)-2 t R(\omega)-\frac{1}{\ell^{2}} 2 t R(k)\right],
\end{array}
$$

where

$$
F^{a}\left(k^{b}\right):=d k^{a}+\epsilon^{a c} \omega k_{c}+\epsilon^{a c} k \omega_{c}+\frac{1}{\ell^{2}} \epsilon^{a c} k k_{c} .
$$

The NR CS action contains three sectors proportional to different arbitrary constants $\tilde{\alpha}_{i}$. The first term is the CS action for the NR Exotic Gravity. The second and third term reproduce the enlarged extended Bargmann gravity with the explicit presence of the $k_{a}$ gauge field. Let us note that the limit $\ell \rightarrow \infty$ in the term proportional to $\tilde{\alpha}_{1}$ reproduces the CS action for the extended Bargmann algebra [25, 26]. On the other hand, the flat limit $\ell \rightarrow \infty$ in the $\tilde{\alpha}_{2}$ term leads us to the CS action for the NR Maxwell algebra [42]. It is important to point out that the term proportional to $\tilde{\alpha}_{1}$ is not the exotic NewtonHooke gravity Lagrangian although it leads to the extended Bargmann gravity Lagrangian in the $\ell \rightarrow \infty$ limit. In particular, the additional gauge fields related to the EEB algebra appearing in the $\tilde{\alpha}_{1}$ term vanish in the flat limit. 
Note that we can express the relativistic gauge fields in terms of the NR ones as follows

$$
\begin{aligned}
& W^{0}=\omega+\frac{s}{2 \xi^{2}}, \\
& W^{a}=\frac{\omega^{a}}{\xi}, \\
& S=\omega-\frac{s}{2 \xi^{2}}, \\
& E^{0}=\xi \tau+\frac{m}{2 \xi}, \\
& E^{a}=e^{a}, \\
& M=\xi \tau-\frac{m}{2 \xi}, \\
& K^{0}=\xi^{2} k+\frac{t}{2}, \\
& K^{a}=\xi k^{a}, \\
& T=\xi^{2} k-\frac{t}{2},
\end{aligned}
$$

such that $A=\tilde{A}$. It is straightforward to show that by using (3.2), (3.11) and (3.16) in the action (2.13), after taking the limit $\xi \rightarrow \infty$ the NR action (3.15) is obtained.

One can notice that the equations of motion from the finite NR action (3.15) reduce to the vanishing of the curvatures (3.14). A completely different situation would occur if the invariant tensor became degenerate during the NR contraction procedure.

\section{Non-relativistic algebras and S-expansion}

In this section we show that the Enlarged Extended Bargmann algebra (3.3) can be alternatively obtained using the $S$-expansion procedure [56]. We show first that the Maxwellian Exotic Bargmann algebra can also be obtained using the $S$-expansion method. In simple words, this method consists in finding a new Lie algebra $\mathfrak{G}=S \times \mathfrak{g}$, by combining the structure constants of a Lie algebra $\mathfrak{g}$ with the elements of a semigroup $S$. Our starting point will be the so-called Nappi-Witten algebra [65, 66] which can be interpreted as a central extension of the homogeneous part of the Galilei algebra. Such Lie algebra can be obtained as a Inönü-Wigner (IW) contraction of a central extension of the Lorentz algebra. In this way, we consider the algebra generated by the Lorentz generator $J_{A}$, and one extra $\mathrm{U}(1)$ generator $Y$.

The contraction process is defined by the identification of the generators defining the [Lorentz] $\oplus u(1)$ algebra, with the Nappi-Witten generators as

$$
J_{0}=\frac{\tilde{J}}{2}+\xi^{2} \tilde{S}, \quad J_{a}=\xi \tilde{G}_{a}, \quad Y=\frac{\tilde{J}}{2}-\xi^{2} \tilde{S},
$$

where $\tilde{J}$ are spatial rotations, $\tilde{G}_{a}$ are Galilean boosts and $\tilde{S}$ is a central charge. The resulting algebra coming from the contraction of the central extension of the Lorentz algebra is

$$
\begin{aligned}
{\left[\tilde{J}, \tilde{G}_{a}\right] } & =\epsilon_{a b} \tilde{G}_{b}, \\
{\left[\tilde{G}_{a}, \tilde{G}_{b}\right] } & =-\epsilon_{a b} \tilde{S} .
\end{aligned}
$$

This algebra, which can be viewed as the Heisenberg algebra with an outher automorphism $J$, will be our original algebra in the expansion process. As we will see, using different Abelian semigroups it is possible to derive different NR algebras.

\subsection{Maxwellian exotic Bargmann algebra}

Let us first obtain the Maxwellian Exotic Bargmann algebra recently introduced in [42]. For this purpose we consider the Nappi-Witten algebra (4.2) and the Abelian semigroup 
$S_{E}^{(2)}=\left\{\lambda_{0}, \lambda_{1}, \lambda_{2}, \lambda_{3}\right\}$, whose multiplication law is given by

\begin{tabular}{|c|cccc|}
\hline$\lambda_{3}$ & $\lambda_{3}$ & $\lambda_{3}$ & $\lambda_{3}$ & $\lambda_{3}$ \\
$\lambda_{2}$ & $\lambda_{2}$ & $\lambda_{3}$ & $\lambda_{3}$ & $\lambda_{3}$ \\
$\lambda_{1}$ & $\lambda_{1}$ & $\lambda_{2}$ & $\lambda_{3}$ & $\lambda_{3}$ \\
$\lambda_{0}$ & $\lambda_{0}$ & $\lambda_{1}$ & $\lambda_{2}$ & $\lambda_{3}$ \\
\hline & $\lambda_{0}$ & $\lambda_{1}$ & $\lambda_{2}$ & $\lambda_{3}$ \\
\hline
\end{tabular}

and where $\lambda_{3} \equiv 0_{S}$ is the zero element of the semigroup such that $0_{S} \lambda_{\alpha}=$ $0_{S}$. After considering a $0_{s}$-reduction, one finds a new algebra whose generators $\left\{\tilde{J}, \tilde{G}_{a}, \tilde{H}, \tilde{P}_{a}, \tilde{Z}, \tilde{Z}_{a} \tilde{S}, \tilde{M}, \tilde{T}\right\}$ are related to the Nappi-Witten ones as

$$
\begin{aligned}
& \tilde{J}=\lambda_{0} \tilde{J}, \quad \tilde{G}_{a}=\lambda_{0} \tilde{G}_{a}, \quad \tilde{S}=\lambda_{0} \tilde{S}, \\
& \tilde{H}=\lambda_{1} \tilde{J}, \quad \tilde{P}_{a}=\lambda_{1} \tilde{G}_{a}, \quad \tilde{M}=\lambda_{1} \tilde{S}, \\
& \tilde{Z}=\lambda_{2} \tilde{J}, \quad \tilde{Z}_{a}=\lambda_{2} \tilde{G}_{a}, \quad \tilde{T}=\lambda_{2} \tilde{S} .
\end{aligned}
$$

Using the multiplication law of the semigroup (4.3) and the Nappi-Witten commutators (4.2), one finds that the expanded generators satisfy the following non-vanishing commutation relations

$$
\begin{aligned}
{\left[\tilde{G}_{a}, \tilde{P}_{b}\right] } & =-\epsilon_{a b} \tilde{M}, & {\left[\tilde{G}_{a}, \tilde{Z}_{b}\right] } & =-\epsilon_{a b} \tilde{T}, \\
{\left[\tilde{H}, \tilde{G}_{a}\right] } & =\epsilon_{a b} \tilde{P}_{b}, & {\left[\tilde{J}, \tilde{Z}_{a}\right] } & =\epsilon_{a b} \tilde{Z}_{b}, \\
{\left[\tilde{J}, \tilde{P}_{a}\right] } & =\epsilon_{a b} \tilde{P}_{b}, & {\left[\tilde{H}, \tilde{P}_{a}\right] } & =\epsilon_{a b} \tilde{Z}_{b}, \\
{\left[\tilde{J}, \tilde{G}_{a}\right] } & =\epsilon_{a b} \tilde{G}_{b}, & {\left[\tilde{P}_{a}, \tilde{P}_{b}\right] } & =-\epsilon_{a b} \tilde{T}, \\
{\left[\tilde{G}_{a}, \tilde{G}_{b}\right] } & =-\epsilon_{a b} \tilde{S}, & {\left[\tilde{Z}, \tilde{G}_{a}\right] } & =\epsilon_{a b} \tilde{Z}_{b}
\end{aligned}
$$

The algebra (4.5) correspond to the Maxwellian Exotic Bargmann algebra and can be obtained as the NR contraction of [Maxwell] $\oplus u(1) \oplus u(1) \oplus u(1)$ algebra. Indeed, as was shown in [42], (4.5) can be obtained from the relativistic Maxwell algebra generated by $\left\{J_{A}, P_{A}, Z_{A}\right\}$ which satisfy

$$
\begin{aligned}
& {\left[J_{A}, J_{B}\right]=\epsilon_{A B C} J^{C},} \\
& {\left[P_{A}, P_{B}\right]=\epsilon_{A B C} Z^{C},} \\
& {\left[J_{A}, Z_{B}\right]=\epsilon_{A B C} Z^{C},} \\
& {\left[J_{A}, P_{B}\right]=\epsilon_{A B C} P^{C},}
\end{aligned}
$$

and three extra $U(1)$ generators $Y_{1}, Y_{2}$ and $Y_{3}$. At the relativistic level, there has been a growing interest in exploring (super)gravity models based on the Maxwell algebra and its generalizations [68-87]. Interestingly a dual version of the three-dimensional Maxwell algebra, known as Hietarinta algebra [88], can be obtained by interchanging the role of the momentum generator $P_{A}$ with the Maxwell gravitational generator $Z_{A}$. In this dual version, 
interesting results have been recently obtained in $[28,89]$ in which a three-dimensional bigravity action has been presented.

The contraction is defined through the following identifications:

$$
\begin{aligned}
& J_{0}=\frac{\tilde{J}}{2}+\xi^{2} \tilde{S}, \quad J_{a}=\xi \tilde{G}_{a}, \quad Y_{2}=\frac{\tilde{J}}{2}-\xi^{2} \tilde{S}, \\
& P_{0}=\frac{\tilde{H}}{2 \xi}+\xi \tilde{M}, \quad P_{a}=\tilde{P}_{a}, \quad Y_{1}=\frac{\tilde{H}}{2 \xi}-\xi \tilde{M}, \\
& Z_{0}=\frac{\tilde{Z}}{2 \xi^{2}}+\tilde{T}, \quad Z_{a}=\frac{\tilde{Z}_{a}}{\xi}, \quad Y_{3}=\frac{\tilde{Z}}{2 \xi^{2}}-\tilde{T} .
\end{aligned}
$$

which is the same identification used in the AdS-Lorentz case. The main difference appears in the absence of the $\ell$ parameter in the relativistic algebra (4.6).

It is worth it to mention that the MEB algebra is obtained by expanding the NappiWittwn algebra using the same semigroup $S_{E}^{(2)}$ used at the relativistic level [90]. As we shall see, such particular feature will appear not only for the MEB algebra. The same behavior appears for infinite-dimensional expanded (super)algebras in which the same expansion relation appearing for finite (super)algebras can be used for infinite-dimensional (super)algebras [91, 92].

\begin{tabular}{|c|c|c|}
\hline $\begin{array}{c}\mathfrak{s o}(2,1) \oplus u(1) \\
\left\{J_{A}, Y\right\} \\
\end{array}$ & $\stackrel{S_{E}^{(2)}}{\longrightarrow}$ & $\begin{array}{c}\text { Maxwell } \oplus u(1)^{3} \\
\left\{J_{A}, P_{A}, Z_{A}, Y_{1}, Y_{2}, Y_{3}\right\}\end{array}$ \\
\hline$\downarrow$ IW contraction & & $\downarrow$ NR limit \\
\hline $\begin{array}{l}\text { Nappi-Witten algebra } \\
\qquad\left\{\tilde{J}, \tilde{G}_{a}, \tilde{S}\right\}\end{array}$ & $\stackrel{S_{E}^{(2)}}{\longrightarrow}$ & $\begin{array}{l}\text { Maxwellian Exotic Bargmann algebra } \\
\qquad\left\{\tilde{J}, \tilde{G}_{a}, \tilde{H}, \tilde{P}_{a}, \tilde{Z}, \tilde{Z}_{a}, \tilde{S}, \tilde{M}, \tilde{T}\right\}\end{array}$ \\
\hline
\end{tabular}

The following diagram summarizes the NR limit as well as the $S$-expansion applied at the relativistic and NR level:

\subsection{Enlargement of the extended Bargmann algebra}

Let us focus now in the NR version of the AdS-Lorentz algebra which is the main topic of the present work. As we have seen, this algebra corresponds to the contraction of the AdS-Lorentz algebra enlarged with three extra U(1) generators, namely the NR limit of [AdS-Lorentz] $\oplus u(1) \oplus u(1) \oplus u(1)$ algebra. An alternative way of deriving this NR algebra is through the $S$-expansion.

As in the previous cases, we first consider the Nappi-Witten algebra (4.2) and a suitable semigroup. For our purpose, we choose the Abelian semigroup $S_{\mathcal{M}}^{(2)}=\left\{\lambda_{0}, \lambda_{1}, \lambda_{2}\right\}$, whose elements satisfy

$$
\lambda_{\alpha} \lambda_{\beta}= \begin{cases}\lambda_{\alpha+\beta} & \text { if } \alpha+\beta \leq 2 \\ \lambda_{\alpha+\beta-2} & \text { if } \alpha+\beta>2\end{cases}
$$

The election of the semigroup is motivated by the fact that the AdS-Lorentz algebra can be obtained as a $S_{\mathcal{M}}^{(2)}$-expansion of the Lorentz algebra in three spacetime dimensions. 
After considering the $S_{\mathcal{M}}^{(2)}$-expansion of the Nappi-Witten algebra one finds an enlargement of the extended Bargmann algebra whose generators $\left\{\tilde{J}, \tilde{G}_{a}, \tilde{H}, \tilde{P}_{a}, \tilde{Z}, \tilde{Z} a, \tilde{S}, \tilde{M}, \tilde{T}\right\}$ satisfy the commutation relations:

$$
\begin{aligned}
& {\left[\tilde{G}_{a}, \tilde{P}_{b}\right]=-\epsilon_{a b} \tilde{M}, \quad\left[\tilde{G}_{a}, \tilde{Z}_{b}\right]=-\epsilon_{a b} \tilde{T}, \quad\left[\tilde{H}, \tilde{Z}_{a}\right]=\frac{1}{\ell^{2}} \epsilon_{a b} \tilde{P}_{b},} \\
& {\left[\tilde{H}, \tilde{G}_{a}\right]=\epsilon_{a b} \tilde{P}_{b}, \quad\left[\tilde{J}, \tilde{Z}_{a}\right]=\epsilon_{a b} \tilde{Z}_{b}, \quad\left[\tilde{Z}_{a}, \tilde{Z}_{b}\right]=-\frac{1}{\ell^{2}} \epsilon_{a b} \tilde{T},} \\
& {\left[\tilde{J}, \tilde{P}_{a}\right]=\epsilon_{a b} \tilde{P}_{b}, \quad\left[\tilde{H}, \tilde{P}_{a}\right]=\epsilon_{a b} \tilde{Z}_{b}, \quad\left[\tilde{P}_{a}, \tilde{Z}_{b}\right]=-\frac{1}{\ell^{2}} \epsilon_{a b} \tilde{M},} \\
& {\left[\tilde{J}, \tilde{G}_{a}\right]=\epsilon_{a b} \tilde{G}_{b}, \quad\left[\tilde{P}_{a}, \tilde{P}_{b}\right]=-\epsilon_{a b} \tilde{T}, \quad\left[\tilde{Z}, \tilde{P}_{a}\right]=\frac{1}{\ell^{2}} \epsilon_{a b} \tilde{P}_{b},} \\
& {\left[\tilde{G}_{a}, \tilde{G}_{b}\right]=-\epsilon_{a b} \tilde{S}, \quad\left[\tilde{Z}, \tilde{G}_{a}\right]=\epsilon_{a b} \tilde{Z}_{b}, \quad\left[\tilde{Z}, \tilde{Z}_{a}\right]=\frac{1}{\ell^{2}} \epsilon_{a b} \tilde{Z}_{b} .}
\end{aligned}
$$

The explicit commutators appears by considering the multiplication law (4.8) and the Nappi-Witten algebra (4.2). In particular, the expanded generators are related to the Nappi-Witten ones as

$$
\begin{aligned}
& \begin{array}{lll}
\tilde{J}=\lambda_{0} \tilde{J}, & \tilde{G}_{a}=\lambda_{0} \tilde{G}_{a}, & \tilde{S}=\lambda_{0} \tilde{S},
\end{array} \\
& \ell \tilde{H}=\lambda_{1} \tilde{J}, \quad \ell \tilde{P}_{a}=\lambda_{1} \tilde{G}_{a}, \quad \ell \tilde{M}=\lambda_{1} \tilde{S}, \\
& \ell^{2} \tilde{Z}=\lambda_{2} \tilde{J}, \quad \ell^{2} \tilde{Z}_{a}=\lambda_{2} \tilde{G}_{a}, \quad \ell^{2} \tilde{T}=\lambda_{2} \tilde{S} .
\end{aligned}
$$

\begin{tabular}{|c|c|c|c|c|}
\hline $\begin{array}{c}\mathfrak{s o}(2,1) \oplus u(1) \\
\left\{J_{A}, Y\right\}\end{array}$ & $\stackrel{S_{\mathcal{M}}^{(2)}}{\longrightarrow}$ & $\begin{array}{c}{[\text { AdS-Lorentz }] \oplus u(1)^{3}} \\
\left\{J_{A}, P_{A}, Z_{A}, Y_{1}, Y_{2}, Y_{3}\right\}\end{array}$ & $\stackrel{\ell \rightarrow \infty}{\longrightarrow}$ & {$[$ Maxwell $] \oplus u(1)^{3}$} \\
\hline$\downarrow$ IW contraction & & $\downarrow$ NR limit & & $\downarrow N R$ limit \\
\hline $\begin{array}{l}\text { Nappi-Witten algebra } \\
\qquad\left\{\tilde{J}, \tilde{G}_{a}, \tilde{S}\right\}\end{array}$ & $\stackrel{S_{\mathcal{M}}^{(2)}}{\longrightarrow}$ & $\begin{array}{c}\text { EEB algebra } \\
\left\{\tilde{J}, \tilde{G}_{a}, \tilde{H}, \tilde{P}_{a}, \tilde{Z}, \tilde{Z}{ }_{a}, \tilde{S}, \tilde{M}, \tilde{T}\right\}\end{array}$ & $\stackrel{\ell \rightarrow \infty}{\longrightarrow}$ & MEB algebra \\
\hline
\end{tabular}

It is interesting to note that the Nappi-Witten algebra is the smallest Lie subalgebra of the EEB algebra containing rotations and boosts. The following diagram summarizes the NR limits, the $S$-expansion applied at the relativistic and NR level and the corresponding flat limits:

A particular advantage of the $S$-expansion procedure is that it allows us to obtain the expanded invariant tensor in terms of the original one [56]. It is well known that the invariant tensor is a crucial ingredient for the construction of a CS action. In particular, the non-vanishing components of the invariant tensor of the Nappi-Witten algebra are given by

$$
\begin{aligned}
\langle\tilde{J} \tilde{S}\rangle & =-1, \\
\left\langle\tilde{G}_{a} \tilde{G}_{b}\right\rangle & =\delta_{a b},
\end{aligned}
$$

Then, considering the definitions of the Theorem VII of [56], we recover the non-vanishing components of the EEB algebra given by (3.9)-(3.10). Let us note that the invariant tensor of the MEB algebra can not only be derived as flat limit $\ell \rightarrow \infty$ of (3.9)-(3.10) but 
can also be obtained from the Nappi-Witten invariant tensor (4.11) considering $S_{E}^{(2)}$ as the relevant semigroup. It would be interesting to extend this methodology to approach new supersymmetric extension of NR gravity theories.

\section{Discussion}

In this work we have presented the NR limit of the relativistic AdS-Lorentz gravity theory. A particular $U(1)$ enlargement of the AdS-Lorentz algebra is considered in order to avoid infinity and degeneracy difficulties. In particular, an enlargement of the extended Bargmann algebra is presented by considering the NR contraction of the [AdS-Lorentz] $\oplus u(1) \oplus u(1) \oplus u(1)$ algebra. Such NR algebra allows to construct a proper finite NR CS action. Interestingly, we have shown that the NR CS gravity theory presented here reproduces the Maxwellian Exotic Bargmann gravity [42] in the limit $\ell \rightarrow \infty$. Furthermore, in such limit the NR CS gravity action contains the Extended Bargmann gravity as a subcase.

We have also studied an alternative method to obtain the EEB and MEB algebra using the $S$-expansion procedure. It is interesting to note that the $S$-expansion method not only provides us with consistent NR algebras but gives us the appropriate central extension of the NR algebras in order to have well defined non-degenerate bilinear form. In particular, at the relativistic level, the same semigroup $S$ gives us the respective relativistic algebras with extra Abelian generators whose presence assures a finite Lagrangian in the NR limit. Let us note that further generalizations of the Galilean algebra have also been obtained through the $S$-expansion method in [93]. On the other hand interesting works have recently appeared in the literature where the Lie algebra expansion method using the Maurer-Cartan equations [94], which is the first report introducing in a general procedure the Lie algebra expansions, has been used to obtain several (super)algebras for NR (super)gravity [95, 96]. It would be worth studying the possibility to obtain the EEB algebra and the MEB one by applying the Lie algebra expansion of [94] to the relativistic AdS-Lorentz and Maxwell algebra, respectively.

It would be interesting to extend the results obtained here at the supersymmetric level. In particular, the Maxwell and AdS-Lorentz supergravity in the CS formalism have been explored recently in $[81,86]$. It would be worth it to explore the extra bosonic field required at the relativistic level to formulate a proper finite NR supergravity action [work in progress].

Another aspect that deserves further investigation is the ultrarelativistic limit of the AdS-Lorentz theory [work in progress]. One could obtain the complete cube, analogously to the ones presented in $[29,97]$, describing the ultrarelativistic, non-relativistic and flat limits for the AdS-Lorentz symmetry. In particular, one could expect to find an Carroll version of the AdS-Lorentz algebra.

\section{Acknowledgments}

The authors would like to thank D. Hidalgo and J. Matulich for valuable comments. This work was supported by the Chilean FONDECYT Projects $N^{\circ} 3170437$ (P.C.) and No3170438 (E.R.). 
Open Access. This article is distributed under the terms of the Creative Commons Attribution License (CC-BY 4.0), which permits any use, distribution and reproduction in any medium, provided the original author(s) and source are credited.

\section{References}

[1] D.T. Son, Toward an AdS/cold atoms correspondence: a geometric realization of the Schrödinger symmetry, Phys. Rev. D 78 (2008) 046003 [arXiv: 0804.3972] [INSPIRE].

[2] K. Balasubramanian and J. McGreevy, Gravity duals for non-relativistic CFTs, Phys. Rev. Lett. 101 (2008) 061601 [arXiv:0804.4053] [INSPIRE].

[3] S. Kachru, X. Liu and M. Mulligan, Gravity duals of Lifshitz-like fixed points, Phys. Rev. D 78 (2008) 106005 [arXiv:0808.1725] [InSPIRE].

[4] A. Bagchi and R. Gopakumar, Galilean conformal algebras and AdS/CFT, JHEP 07 (2009) 037 [arXiv: 0902.1385] [INSPIRE].

[5] A. Bagchi, R. Gopakumar, I. Mandal and A. Miwa, GCA in 2d, JHEP 08 (2010) 004 [arXiv:0912.1090] [INSPIRE].

[6] M.H. Christensen, J. Hartong, N.A. Obers and B. Rollier, Torsional Newton-Cartan geometry and Lifshitz holography, Phys. Rev. D 89 (2014) 061901 [arXiv:1311.4794] [InSPIRE].

[7] M.H. Christensen, J. Hartong, N.A. Obers and B. Rollier, Boundary stress-energy tensor and Newton-Cartan geometry in Lifshitz holography, JHEP 01 (2014) 057 [arXiv:1311.6471] [INSPIRE].

[8] M. Taylor, Lifshitz holography, Class. Quant. Grav. 33 (2016) 033001 [arXiv:1512.03554] [INSPIRE].

[9] D.T. Son, Newton-Cartan geometry and the quantum Hall effect, arXiv:1306.0638 [INSPIRE].

[10] C. Hoyos and D.T. Son, Hall viscosity and electromagnetic response, Phys. Rev. Lett. 108 (2012) 066805 [arXiv:1109.2651] [INSPIRE].

[11] M. Geracie, K. Prabhu and M.M. Roberts, Curved non-relativistic spacetimes, Newtonian gravitation and massive matter, J. Math. Phys. 56 (2015) 103505 [arXiv: 1503.02682] [INSPIRE].

[12] A. Gromov, K. Jensen and A.G. Abanov, Boundary effective action for quantum Hall states, Phys. Rev. Lett. 116 (2016) 126802 [arXiv:1506.07171] [INSPIRE].

[13] C. Duval and H.P. Kunzle, Minimal gravitational coupling in the Newtonian theory and the covariant Schrödinger equation, Gen. Rel. Grav. 16 (1984) 333 [INSPIRE].

[14] C. Duval, G. Burdet, H.P. Kunzle and M. Perrin, Bargmann structures and Newton-Cartan theory, Phys. Rev. D 31 (1985) 1841 [INSPIRE].

[15] C. Duval, G.W. Gibbons and P. Horvathy, Celestial mechanics, conformal structures and gravitational waves, Phys. Rev. D 43 (1991) 3907 [hep-th/0512188] [INSPIRE].

[16] C. Duval, On Galileian isometries, Class. Quant. Grav. 10 (1993) 2217 [arXiv:0903.1641] [INSPIRE]. 
[17] R. De Pietri, L. Lusanna and M. Pauri, Standard and generalized Newtonian gravities as 'gauge' theories of the extended Galilei group. I. The standard theory, Class. Quant. Grav. 12 (1995) 219 [gr-qc/9405046] [INSPIRE].

[18] R. De Pietri, L. Lusanna and M. Pauri, Standard and generalized Newtonian gravities as 'gauge' theories of the extended Galilei group. II. Dynamical three space theories, Class. Quant. Grav. 12 (1995) 255 [gr-qc/9405047] [INSPIRE].

[19] P. Hořava, Quantum gravity at a Lifshitz point, Phys. Rev. D 79 (2009) 084008 [arXiv:0901.3775] [INSPIRE].

[20] C. Duval and P.A. Horvathy, Non-relativistic conformal symmetries and Newton-Cartan structures, J. Phys. A 42 (2009) 465206 [arXiv:0904.0531] [INSPIRE].

[21] G. Papageorgiou and B.J. Schroers, Galilean quantum gravity with cosmological constant and the extended q-Heisenberg algebra, JHEP 11 (2010) 020 [arXiv:1008.0279] [INSPIRE].

[22] R. Andringa, E. Bergshoeff, S. Panda and M. de Roo, Newtonian gravity and the Bargmann algebra, Class. Quant. Grav. 28 (2011) 105011 [arXiv:1011.1145] [INSPIRE].

[23] R. Andringa, E. Bergshoeff, J. Gomis and M. de Roo, 'Stringy' Newton-Cartan gravity, Class. Quant. Grav. 29 (2012) 235020 [arXiv:1206.5176] [INSPIRE].

[24] E. Bergshoeff, J. Rosseel and T. Zojer, Newton-Cartan (super)gravity as a non-relativistic limit, Class. Quant. Grav. 32 (2015) 205003 [arXiv: 1505.02095] [INSPIRE].

[25] E.A. Bergshoeff and J. Rosseel, Three-dimensional extended Bargmann supergravity, Phys. Rev. Lett. 116 (2016) 251601 [arXiv: 1604.08042] [INSPIRE].

[26] J. Hartong, Y. Lei and N.A. Obers, Nonrelativistic Chern-Simons theories and three-dimensional Hořava-Lifshitz gravity, Phys. Rev. D 94 (2016) 065027 [arXiv: 1604.08054] [INSPIRE].

[27] E. Bergshoeff, A. Chatzistavrakidis, L. Romano and J. Rosseel, Newton-Cartan gravity and torsion, JHEP 10 (2017) 194 [arXiv:1708.05414] [INSPIRE].

[28] D. Chernyavsky and D. Sorokin, Three-dimensional (higher-spin) gravities with extended Schrödinger and l-conformal Galilean symmetries, arXiv:1905.13154 [INSPIRE].

[29] H. Bacry and J. Levy-Leblond, Possible kinematics, J. Math. Phys. 9 (1968) 1605 [INSPIRE].

[30] H. Bacry and J. Nuyts, Classification of ten-dimensional kinematical groups with space isotropy, J. Math. Phys. 27 (1986) 2455 [INSPIRE].

[31] R. Aldrovandi, A.L. Barbosa, L.C.B. Crispino and J.G. Pereira, Non-relativistic spacetimes with cosmological constant, Class. Quant. Grav. 16 (1999) 495 [gr-qc/9801100] [INSPIRE].

[32] O. Arratia, M.A. Martin and M.A. Olmo, Classical systems and representations of $(2+1)$ Newton-Hooke symmetries, math-ph/9903013.

[33] Y.-H. Gao, Symmetries, matrices and de Sitter gravity, Conf. Proc. C 0208124 (2002) 271 [hep-th/0107067] [INSPIRE].

[34] G.W. Gibbons and C.E. Patricot, Newton-Hooke space-times, Hpp waves and the cosmological constant, Class. Quant. Grav. 20 (2003) 5225 [hep-th/0308200] [INSPIRE].

[35] J. Brugues, J. Gomis and K. Kamimura, Newton-Hooke algebras, non-relativistic branes and generalized pp-wave metrics, Phys. Rev. D 73 (2006) 085011 [hep-th/0603023] [INSPIRE]. 
[36] P.D. Alvarez, J. Gomis, K. Kamimura and M.S. Plyushchay, $(2+1) D$ exotic Newton-Hooke symmetry, duality and projective phase, Annals Phys. 322 (2007) 1556 [hep-th/0702014] [INSPIRE].

[37] E. Witten, $(2+1)$-dimensional gravity as an exactly soluble system, Nucl. Phys. B 311 (1988) 46 [inSPIRE].

[38] A. Achucarro and P.K. Townsend, A Chern-Simons action for three-dimensional anti-de Sitter supergravity theories, Phys. Lett. B 180 (1986) 89 [INSPIRE].

[39] J. Zanelli, Lecture notes on Chern-Simons (super-)gravities. Second edition (February 2008), in Proceedings, $7^{\text {th }}$ Mexican Workshop on Particles and Fields (MWPF 1999), Merida, Mexico, 10-17 November 1999 [hep-th/0502193] [INSPIRE].

[40] J. Gomis and H. Ooguri, Nonrelativistic closed string theory, J. Math. Phys. 42 (2001) 3127 [hep-th/0009181] [INSPIRE].

[41] A. Barducci, R. Casalbuoni and J. Gomis, Non-relativistic spinning particle in a Newton-Cartan background, JHEP 01 (2018) 002 [arXiv:1710.10970] [INSPIRE].

[42] L. Avilés, E. Frodden, J. Gomis, D. Hidalgo and J. Zanelli, Non-relativistic Maxwell Chern-Simons gravity, JHEP 05 (2018) 047 [arXiv: 1802.08453] [INSPIRE].

[43] H. Bacry, P. Combe and J.L. Richard, Group-theoretical analysis of elementary particles in an external electromagnetic field. 1. The relativistic particle in a constant and uniform field, Nuovo Cim. A 67 (1970) 267 [inSPIRE].

[44] R. Schrader, The Maxwell group and the quantum theory of particles in classical homogeneous electromagnetic fields, Fortsch. Phys. 20 (1972) 701 [InSPIRE].

[45] J. Gomis and A. Kleinschmidt, On free Lie algebras and particles in electro-magnetic fields, JHEP 07 (2017) 085 [arXiv: 1705.05854] [INSPIRE].

[46] D.V. Soroka and V.A. Soroka, Semi-simple extension of the (super)Poincaré algebra, Adv. High Energy Phys. 2009 (2009) 234147 [hep-th/0605251] [INSPIRE].

[47] J. Gomis, K. Kamimura and J. Lukierski, Deformations of Maxwell algebra and their dynamical realizations, JHEP 08 (2009) 039 [arXiv:0906.4464] [INSPIRE].

[48] P.K. Concha, R. Durka, C. Inostroza, N. Merino and E.K. Rodríguez, Pure Lovelock gravity and Chern-Simons theory, Phys. Rev. D 94 (2016) 024055 [arXiv:1603.09424] [InSPIRE].

[49] P.K. Concha, N. Merino and E.K. Rodríguez, Lovelock gravities from Born-Infeld gravity theory, Phys. Lett. B 765 (2017) 395 [arXiv: 1606. 07083] [INSPIRE].

[50] P. Concha and E. Rodríguez, Generalized pure Lovelock gravity, Phys. Lett. B 774 (2017) 616 [arXiv:1708.08827] [INSPIRE].

[51] P.K. Concha, E.K. Rodríguez and P. Salgado, Generalized supersymmetric cosmological term in $N=1$ supergravity, JHEP 08 (2015) 009 [arXiv:1504.01898] [INSPIRE].

[52] M.C. Ipinza, P.K. Concha, L. Ravera and E.K. Rodríguez, On the supersymmetric extension of Gauss-Bonnet like gravity, JHEP 09 (2016) 007 [arXiv:1607.00373] [INSPIRE].

[53] A. Banaudi and L. Ravera, Generalized AdS-Lorentz deformed supergravity on a manifold with boundary, Eur. Phys. J. Plus 133 (2018) 514 [arXiv: 1803.08738] [InSPIRE].

[54] D.M. Peñafiel and L. Ravera, Generalized cosmological term in $D=4$ supergravity from a new AdS-Lorentz superalgebra, Eur. Phys. J. C 78 (2018) 945 [arXiv:1807.07673] [INSPIRE]. 
[55] P. Concha, N. Merino, E. Rodríguez, P. Salgado-Rebolledó and O. Valdivia, Semi-simple enlargement of the $\mathfrak{b m s}_{3}$ algebra from a $\mathfrak{s o}(2,2) \oplus \mathfrak{s o}(2,1)$ Chern-Simons theory, JHEP 02 (2019) 002 [arXiv: 1810.12256] [INSPIRE].

[56] F. Izaurieta, E. Rodriguez and P. Salgado, Expanding Lie (super)algebras through Abelian semigroups, J. Math. Phys. 47 (2006) 123512 [hep-th/0606215] [INSPIRE].

[57] R. Caroca, I. Kondrashuk, N. Merino and F. Nadal, Bianchi spaces and their three-dimensional isometries as $S$-expansions of two-dimensional isometries, J. Phys. A 46 (2013) 225201 [arXiv:1104.3541] [INSPIRE].

[58] L. Andrianopoli, N. Merino, F. Nadal and M. Trigiante, General properties of the expansion methods of Lie algebras, J. Phys. A 46 (2013) 365204 [arXiv:1308.4832] [InSPIRE].

[59] D.M. Peñafiel and P. Salgado-Rebolledó, Non-relativistic symmetries in three space-time dimensions and the Nappi-Witten algebra, arXiv:1906.02161 [INSPIRE].

[60] J. Diaz et al., A generalized action for $(2+1)$-dimensional Chern-Simons gravity, J. Phys. A 45 (2012) 255207 [arXiv:1311.2215] [inSPIRE].

[61] P. Salgado and S. Salgado, $\mathfrak{s o}(D-1,1) \otimes \mathfrak{s o}(D-1,2)$ algebras and gravity, Phys. Lett. B 728 (2014) 5 [inSPIRE].

[62] S. Hoseinzadeh and A. Rezaei-Aghdam, $(2+1)$-dimensional gravity from Maxwell and semisimple extension of the Poincaré gauge symmetric models, Phys. Rev. D 90 (2014) 084008 [arXiv: 1402.0320] [INSPIRE].

[63] P. Salgado, R.J. Szabo and O. Valdivia, Topological gravity and transgression holography, Phys. Rev. D 89 (2014) 084077 [arXiv: 1401.3653] [INSPIRE].

[64] P. Concha, N. Merino, O. Mišković, E. Rodríguez, P. Salgado-Rebolledó and O. Valdivia, Asymptotic symmetries of three-dimensional Chern-Simons gravity for the Maxwell algebra, JHEP 10 (2018) 079 [arXiv:1805.08834] [INSPIRE].

[65] C.R. Nappi and E. Witten, A WZW model based on a nonsemisimple group, Phys. Rev. Lett. 71 (1993) 3751 [hep-th/9310112] [INSPIRE].

[66] J.M. Figueroa-O'Farrill and S. Stanciu, More D-branes in the Nappi-Witten background, JHEP 01 (2000) 024 [hep-th/9909164] [INSPIRE].

[67] O. Fierro, F. Izaurieta, P. Salgado and O. Valdivia, Minimal AdS-Lorentz supergravity in three-dimensions, Phys. Lett. B 788 (2019) 198 [arXiv:1401.3697] [INSPIRE].

[68] J.A. de Azcarraga, K. Kamimura and J. Lukierski, Generalized cosmological term from Maxwell symmetries, Phys. Rev. D 83 (2011) 124036 [arXiv:1012.4402] [INSPIRE].

[69] R. Durka, J. Kowalski-Glikman and M. Szczachor, Gauged AdS-Maxwell algebra and gravity, Mod. Phys. Lett. A 26 (2011) 2689 [arXiv:1107.4728] [INSPIRE].

[70] J.A. de Azcarraga, K. Kamimura and J. Lukierski, Maxwell symmetries and some applications, Int. J. Mod. Phys. Conf. Ser. 23 (2013) 01160 [arXiv:1201.2850] [INSPIRE].

[71] P.K. Concha, D.M. Peñafiel, E.K. Rodríguez and P. Salgado, Even-dimensional general relativity from Born-Infeld gravity, Phys. Lett. B 725 (2013) 419 [arXiv:1309.0062] [INSPIRE].

[72] P.K. Concha, D.M. Penafiel, E.K. Rodriguez and P. Salgado, Chern-Simons and Born-Infeld gravity theories and Maxwell algebras type, Eur. Phys. J. C 74 (2014) 2741 [arXiv: 1402.0023] [INSPIRE]. 
[73] S. Bonanos, J. Gomis, K. Kamimura and J. Lukierski, Maxwell superalgebra and superparticle in constant gauge backgrounds, Phys. Rev. Lett. 104 (2010) 090401 [arXiv:0911.5072] [INSPIRE].

[74] J.A. de Azcarraga and J.M. Izquierdo, Minimal D $=4$ supergravity from the super-Maxwell algebra, Nucl. Phys. B 885 (2014) 34 [arXiv:1403.4128] [InSPIRE].

[75] P.K. Concha and E.K. Rodríguez, $N=1$ supergravity and Maxwell superalgebras, JHEP 09 (2014) 090 [arXiv:1407.4635] [INSPIRE].

[76] P.K. Concha, O. Fierro, E.K. Rodríguez and P. Salgado, Chern-Simons supergravity in D $=3$ and Maxwell superalgebra, Phys. Lett. B 750 (2015) 117 [arXiv: 1507.02335] [INSPIRE].

[77] P.K. Concha, O. Fierro and E.K. Rodríguez, Inönü-Wigner contraction and D=2+1 supergravity, Eur. Phys. J. C 77 (2017) 48 [arXiv:1611.05018] [INSPIRE].

[78] D.M. Peñafiel and L. Ravera, On the hidden Maxwell superalgebra underlying $D=4$ supergravity, Fortsch. Phys. 65 (2017) 1700005 [arXiv: 1701.04234] [INSPIRE].

[79] R. Caroca, P. Concha, O. Fierro, E. Rodríguez and P. Salgado-Rebolledó, Generalized Chern-Simons higher-spin gravity theories in three dimensions, Nucl. Phys. B 934 (2018) 240 [arXiv:1712.09975] [INSPIRE].

[80] L. Ravera, Hidden role of Maxwell superalgebras in the free differential algebras of $D=4$ and $D=11$ supergravity, Eur. Phys. J. C 78 (2018) 211 [arXiv:1801.08860] [INSPIRE].

[81] P. Concha, D.M. Peñafiel and E. Rodríguez, On the Maxwell supergravity and flat limit in $2+1$ dimensions, Phys. Lett. B 785 (2018) 247 [arXiv:1807.00194] [InSPIRE].

[82] P. Concha, L. Ravera and E. Rodríguez, On the supersymmetry invariance of flat supergravity with boundary, JHEP 01 (2019) 192 [arXiv: 1809.07871] [INSPIRE].

[83] J. Gomis, A. Kleinschmidt and J. Palmkvist, Symmetries of M-theory and free Lie superalgebras, JHEP 03 (2019) 160 [arXiv:1809.09171] [INSPIRE].

[84] S. Kibaroğlu, M. Şenay and O. Cebecioğlu, $D=4$ topological gravity from gauging the Maxwell-special-affine group, Mod. Phys. Lett. A 34 (2019) 1950016 [arXiv:1810.01635] [INSPIRE].

[85] S. Kibaroğlu and O. Cebecioğlu, $D=4$ supergravity from the Maxwell-Weyl superalgebra, arXiv: 1812.09861 [INSPIRE].

[86] P. Concha, $N$-extended Maxwell supergravities as Chern-Simons theories in three spacetime dimensions, Phys. Lett. B 792 (2019) 290 [arXiv:1903.03081] [InSPIRE].

[87] P. Salgado-Rebolledó, The Maxwell group in $2+1$ dimensions and its infinite-dimensional enhancements, arXiv: 1905.09421 [INSPIRE].

[88] J. Hietarinta, Supersymmetry generators of arbitrary spin, Phys. Rev. D 13 (1976) 838 [INSPIRE].

[89] S. Bansal and D. Sorokin, Can Chern-Simons or Rarita-Schwinger be a Volkov-Akulov Goldstone?, JHEP 07 (2018) 106 [arXiv:1806.05945] [INSPIRE].

[90] P.K. Concha and E.K. Rodríguez, Maxwell superalgebras and Abelian semigroup expansion, Nucl. Phys. B 886 (2014) 1128 [arXiv: 1405.1334] [InSPIRE].

[91] R. Caroca, P. Concha, E. Rodríguez and P. Salgado-Rebolledó, Generalizing the $\mathfrak{b m s}_{3}$ and 2D-conformal algebras by expanding the Virasoro algebra, Eur. Phys. J. C 78 (2018) 262 [arXiv: 1707.07209] [INSPIRE]. 
[92] R. Caroca, P. Concha, O. Fierro and E. Rodríguez, Three-dimensional Poincaré supergravity and $N$-extended supersymmetric BMS 3 algebra, Phys. Lett. B 792 (2019) 93 [arXiv: 1812.05065] [INSPIRE].

[93] N. González, G. Rubio, P. Salgado and S. Salgado, Generalized Galilean algebras and Newtonian gravity, Phys. Lett. B 755 (2016) 433 [arXiv: 1604.06313] [INSPIRE].

[94] J.A. de Azcarraga, J.M. Izquierdo, M. Picón and O. Varela, Generating Lie and gauge free differential (super)algebras by expanding Maurer-Cartan forms and Chern-Simons supergravity, Nucl. Phys. B 662 (2003) 185 [hep-th/0212347] [INSPIRE].

[95] E. Bergshoeff, J.M. Izquierdo, T. Ortín and L. Romano, Lie algebra expansions and actions for non-relativistic gravity, arXiv: 1904.08304 [INSPIRE].

[96] J.A. de Azcárraga, D. Gútiez and J.M. Izquierdo, Extended D $=3$ Bargmann supergravity from a Lie algebra expansion, arXiv:1904.12786 [INSPIRE].

[97] J. Matulich, S. Prohazka and J. Salzer, Limits of three-dimensional gravity and metric kinematical Lie algebras in any dimension, arXiv:1903.09165 [INSPIRE]. 